\title{
A Cluster-based Localization Method using RSSI for Heterogeneous Wireless Sensor Networks
}

\author{
Chen Zhong, Jens Eliasson, Henrik Mäkitaavola \\ Dept. of Computer Science and Electrical Engineering \\ Luleå University of Technology \\ S-971 87 Luleå, Sweden \\ Email: \{chen.zhong, jens.eliasson, henrik.makitaavola\}@ltu.se
}

\author{
Fan Zhang \\ Dept. of Logistics Engineering \\ Wuhan University of Technology \\ 430063, WuHan, P.R. China \\ Email: ailexy@whut.edu.cn
}

\begin{abstract}
In this paper, we investigate the performance of cluster-based localization using received signal strength indicator (RSSI). The proposed solution is designed to meet the requirements of monitoring of firefighters or similar applications. The empirical relationship between signal strength and distance is determined using experiment data. One of the most popular localization algorithms found today, Min-Max, is used for our testbeds. Our solution is implemented in TinyOS and experimentally evaluated on a Mulle v5.2 IEEE 802.15.4 platform. The aim of our research is to develop a heterogeneous wireless sensor network consisting of inter connected body area networks, or clusters. Using localization, the network's robustness and reliability, as well as the safety of its users, can be improved.
\end{abstract}

Index Terms-Localization, RSSI, WSN, IEEE 802.15.4

\section{INTRODUCTION}

Localization is one of the most important services in the field of wireless sensor networks (WSNs). Firstly, localization is necessary when implementing some applications, such as water quality monitoring and indoor air quality monitoring [1], as it might be inadequate to only collect sensor data without the knowledge of its corresponding location. Secondly, knowing the relative position of sensor nodes allows the use of geography-based routing protocols, which can improve the performance of a network in terms of reliability and low power consumption [2]. The Global Positioning System is a very popular location estimation system, however it only works outdoors where it can receive the satellites' signals. However, many WSN applications are running indoors which prohibits the usage of GPS. Alternatively, methods using other technologies, e.g. ultrasound [3], can achieve high accuracy in positioning, but each device adds to the sensor node size, cost, and energy consumption (which is one of the most critical issues in WSNs). Comparing with mentioned localization approaches, the advantages of using received signal strength indicator (RSSI) based localization are quite apparent. Technically, it can be used both inside of a building or outdoors. Furthermore, no additional hardware is required since wireless sensor nodes are equipped with radio transceivers. The challenges of localization based on RSSI are mainly due to its unpredictable propagation, especially in indoor environment. The reflections of a signal against walls, floors and ceilings may result in severe multi-path interference at the receiving antenna, as shown by Zanca et al. [4], with increased errors as a result.

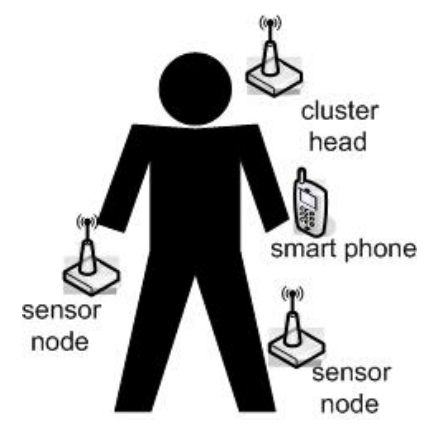

Fig. 1. Body Area Network overview

The target application is monitoring of firefighters in order to improve their safety in hazardous working environments. The goal of our research is to develop an architecture for heterogeneous wireless sensor network consisting of a number of inter connected small body area (sensor) networks (Fig. 1). The proposed localization approach will be used for improving overall network connectivity as well as locating firefighters in need of assistance.

The rest of the paper is organized as follows: Section II discusses related work. The application scenario and requirements are presented in Section III. Experiments and results are described in Section VI. In the last section, conclusions are drawn and future work are outlined.

\section{RELATED WORK}

Considerable research has been carried out in the area of localization based on RSSI. Stoyanova et al. [5] performed a theoretical analysis and experimental evaluation of impact factors on received signal strength (RSS) accuracy, and Sugano et al. [6] implemented an indoor localization system on a Ubiquitous Device. Besides the environment where the sensor nodes are deployed, radio characteristics, such as antenna type and size, channel frequency, modulation and transmit power, can influence the signal propagation drastically as well. For instance, the Ubiquitous Device uses a relatively large antenna which improves the radio performance, but make the 


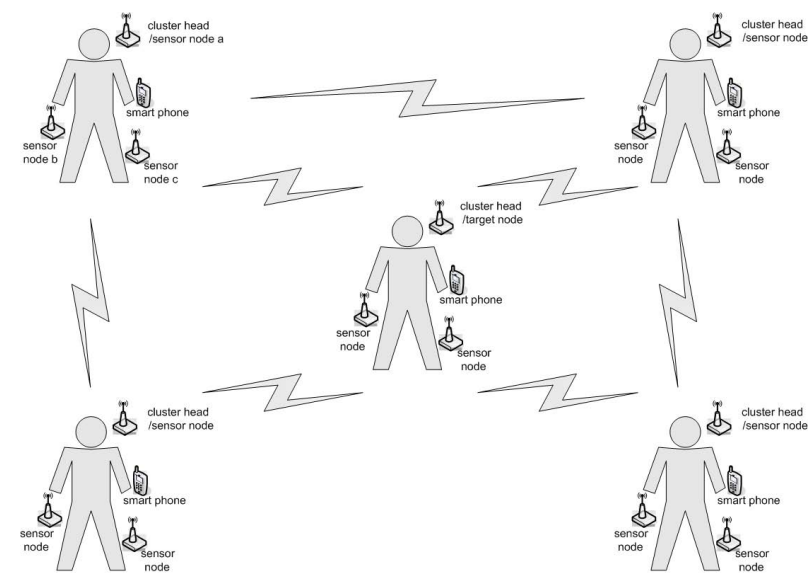

Fig. 2. Cluster-based localization in a heterogeneous wireless sensor network

deployment of sensor nodes inconvenient in the real world because of its large size.

In [7] Elnahraway et al. tried to experimentally demonstrate the limits of a variety of RSS-based localization algorithms. They used commodity IEEE 802.11 technology and concluded that the limitations are fundamental. Comparing with that, Zanca et al. [4] investigated the real-world performance of some well known localization algorithms using the IEEE 802.15.4 standard. According to their findings, RSSI-based localization in indoor environments presents severe limitations, but they also suggested that an accurate radio channel model might alleviate the problems mainly introduced by the environment.

Generally, current research suggests that RSS-based localization is a promising technique but is currently suffering from high inaccuracy. However, this situation can be improved by analyzing a specific application scenario, and adjusting a localization algorithm towards its specific requirements. Using this approach it is possible to devise a localization method which takes advantage of specific characteristics that general methods can not.

One of the most popular localization algorithms is the MinMax [8]. Compared to other algorithms, it is not complicated to implement. Complex functionalities running on a sensor node, such as localization, can provide a significant contribution to higher level applications. However, a sensor node typically has very limited resources in terms of computation power, memory, and energy. Therefore, it is crucial that any function can be implemented using small resources as possible. In [9], localization based on the maximum likelihood (ML) method has been presented. The ML estimation of a target is obtained by using the minimum mean square error (MMSE). The main idea of MMSE is that if the distance between an anchor and the target is known, then the target must lay on a circle centered at the anchor and with a radius equaling to the distance. At least three distances with different anchors are needed.

The localization algorithm investigated in this paper is the Min-Max due to its relatively good performance and low resource requirements. For each anchor, it will construct a bounding square box. The center is the anchor itself and the edges is the double of the estimated distance with the target. Assume there are three anchors which are distributed in 2D manner. As a result, three corresponding boxes and the intersection of these boxes can be obtained. The position of a target is defined as the center of intersection of the boxes. In [4], Zanca et al. compared the 2D Min-Max with other algorithm, such as ML. Their research was however general and thus lacking application characteristics. In [10], Parker et al. used the Min-Max localization for vehicle positioning based on the IEEE $802.11 \mathrm{p}$ standard. They used the algorithm in pure $2 \mathrm{D}$ experiments.

\section{APPLICATION SCENARIO AND REQUIREMENTS}

The goal of this project is to increase safety for personnel, e.g. police officers, assault teams, and firefighters, working in hazardous environments. In order to achieve this goal, it is necessary to be able to remotely monitor the personnel participating in an operation. One main issue to deal with is that this types of operations are highly mobile, e.g. taking place in forests, on roads, in buildings, and in tunnels. Therefore, it is important that the communication infrastructure is wireless and has extremely good coverage. Today, the only means of achieving this is by using commonly available infrastructures, such as the mobile telephone network ( $3 \mathrm{G}$, GPRS) and the Internet. One approach is to equip every sensor node with a GPRS module. This is however an expensive solution. Another issue is that it is desired that each user can be equipped several sensors in order to collect different types of information. The use of cables is prohibited due to the fact that cables can conduct heat and might interfere with the user's movement. The approach adopted here therefore to use several sensor nodes communicating wirelessly with each other and the gateway using low cost, short range radios. The gateway can be either a PDA, mobile phone, or some specialized device. All nodes located on a user form a body area network (BAN, cluster), which collect and forward sensor data to the gateway. Fig. 1 shows one example of a BAN consisting of three sensor nodes (one node also acts as cluster head) and mobile phone gateway. Another advantage when using a mobile phone is that a user's standard mobile phone can be reused as infrastructure access point. This approach eliminates the need to carry additional hardware and reduces the cost. Also, the possibility when the mobile phone connection in a cluster is lost is considered. In such circumstance, the BAN will try to route its sensor data to another BAN in its vicinity. Then, the network topology will become a mesh network. Localization can assist such routing when mobile phone failure occur and thus improve the network reliability.

Sensor nodes within a BAN can acquire information about its user, such as when a user falls down, and position, temperature, pulse, posture, etc. When an anomaly is detected, an alarm message must be sent to the network's infrastructure and collected at a command center. It might be vital for the command center to know the location for each person. If 
an emergency would happen to a person, actions to assist this person should be taken as soon as possible. As a result, using localization can accelerate the search procedure and save time for following measures. Thus, it is motivated to explore the indoor localization. One significant characteristic for this application is that a cluster which contains several sensor nodes should be located. Fig. 2 shows an example of a wireless sensor network consisting of five body area networks (clusters). Every sensor node within the same cluster can estimate the distance between itself and neighboring clusters. However, usually not all sensor nodes in a cluster can estimate the distance precisely due to the degradation of the signal propagation due to the environment. It is even worse that the nodes might report quite different distances although actually they are near each other. The contribution to this issue can be the propagation of signal and the orientation of the antenna. In the experiments considered in this paper it was found that the latter contribution was one of the most critical challenges. For instance in Fig. 2, if the orientation of anchor nodes, $a, b, c$ is different, the RSSIs between the Target node in cluster 4 and the anchors, $a, b, c$ in cluster 1 , can be rather dissimilar. The focus is not on the relationship between the antenna orientation and received signal strength since the users are mobile and antenna orientation will constantly change. Therefore, the highest RSSI out of all sensor nodes' RSSIs in a cluster was chosen to reflect the distance between the target node and other clusters. The performance of the localization method using different configurations, such as, the number of anchors in a cluster, and the used amount of RSSI(s) from different anchor(s) in the same reference point (cluster) were studied.

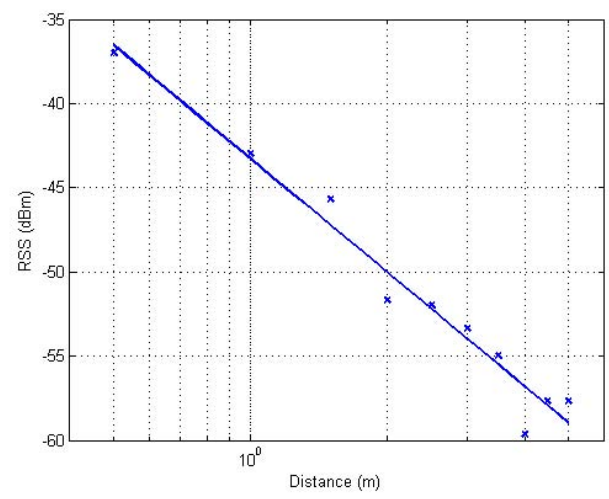

Fig. 3. $10 \mathrm{~cm}$ above the ground

\section{RSSI-DiSTANCE CHARACTERIZATION}

Before performing localization experiments, it was essential to find a suitable model for the relationship between the measured RSSI value and the distance between nodes. In order to achieve this, a number of packets were sent when the target and anchor nodes were separated with different distances. The average of all RSSI values were taken as the measured RSSI value for a certain distance. These experiments are performed

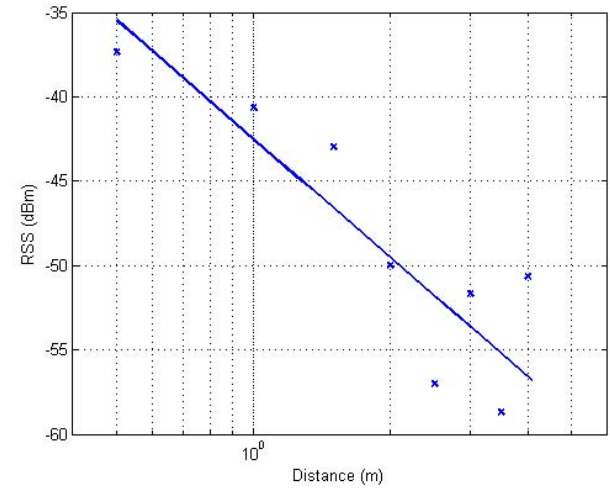

Fig. $4 . \quad 80 \mathrm{~cm}$ above the ground

in different heights above the ground, $0.1 \mathrm{~m}$ and $0.8 \mathrm{~m}$. Results are shown in Fig. 3 and Fig. 4 respectively.

Based on obtained experimental data and using logarithmic curve fitting, the relationships between measured signal strength $(\mathrm{dBm})$ and distance $(\mathrm{m})$ can be expressed in the following way:

$10 \mathrm{~cm}$ above the ground

$$
s=-9.73681 \ln d-43.3088
$$

$80 \mathrm{~cm}$ above the ground

$$
s=-10.14731 \ln d-42.5258
$$

The formulas are plotted as a straight line in logarithmic coordinates. As it can be seen in Fig. 3 and Fig. 4, the RSS values distribute quite close to the fitting line in the occasion of $0.1 \mathrm{~m}$ above the ground, while in comparison, the RSS values spread slightly around their fitting line in the $0.8 \mathrm{~m}$ case. In the following sections, these relationships are used to estimate the distances from the target to all anchor nodes.

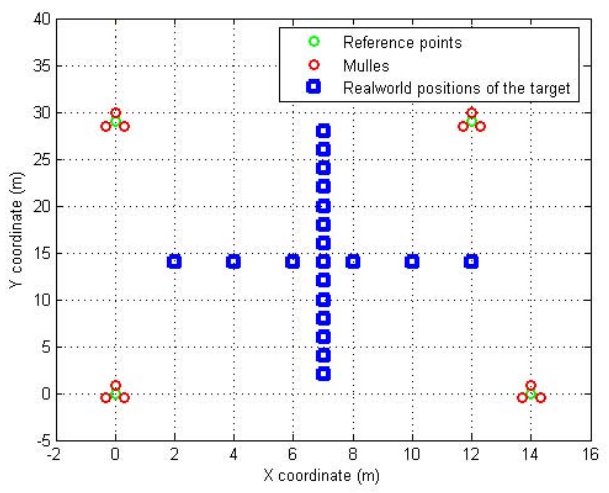

Fig. 5. Test bed

\section{Testbed}

The experiments have been performed in a specially designed testbed. The testbed is based on a coordinate system consisting of four reference points. The $\mathrm{x}, \mathrm{y}$ coordinates for 
each test position and anchor are depicted in Fig. 5. Near each reference point, we uniformly deployed three sensor nodes as anchors. Nearby anchors constitute a cluster. The fourteen vertical and six horizontal small squares stand for the positions where the target node is placed and at each of these location, it broadcasts ten localization request packets. On the receiver side, the anchors might receive all these ten packets, and thus retrieve the RSSIs for the calculation of corresponding location. In order to investigate the influence of aptitude above the ground in positioning, each time, the target was placed in several different heights above the ground. Three setup positions (heights) of target node on a body were emulated, head, belly and knee. The heights ( $\mathrm{Z}$ axis) assumed for these three positions are $2 \mathrm{~m}, 1 \mathrm{~m}$ and $0.5 \mathrm{~m}$ respectively.

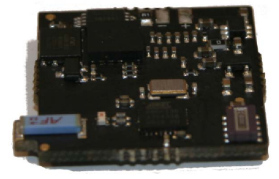

Fig. 6. Mulle v5.2, IEEE 802.15.4 sensor node

\section{EXPERIMENTAL RESULTS}

Our localization solution is evaluated on the Mulle v5.2 IEEE 802.15.4 platform from Eistec $\mathrm{AB}$ [11], which uses an Atmel AT86RF230 [12] transceiver. The experimental results are obtained based on running Min-Max algorithm. The influence of RSS-Distance model's parameters, the number of localization request packets and the number of anchors in a cluster on positioning accuracy were analyzed respectively. To evaluate the over-all performance among different configurations, some estimation error means were also calculated.

- Estimation Error - RSS-Distance Model First of all, the effect of RSS-Distance model's parameters to estimation error is studied. As mentioned, two models to characterize the relationship between RSS and distance at two different heights above ground $(0.1 \mathrm{~m}$ and $0.8 \mathrm{~m})$ were derived. However, in Fig. 7, it is shown that the performance of localization seems to not rely on the model. For different number (one, two and three) of anchor nodes in each reference point (cluster) used, the error distributions are nearly the same for different RSS-Distance models. Thus the localization system is almost independent of models and the $0.8 \mathrm{~m}$ above ground RSS-Distance model will be adopted for further analysis.

- Estimation Error - Localization Request Packet Used Amount Secondly, the influence of the number of RSSI measurement packets used for localization is investigated. Originally, there are ten packets broadcasted by a target. Due to environmental interference to signal propagation, some packets are lost, and in a certain test, only six packets can be received by a anchor node. As a result,

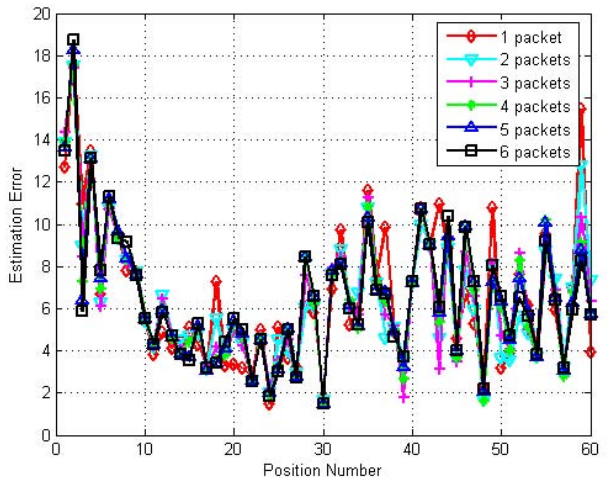

Fig. 8. Three anchors in each reference point, different number of RSSI measurement packets used in each localization experiment

a maximum of six RSSI measurement packets are available to compute the mean value of estimated distance. Therefore, the influence of packet number to estimation error can be depicted by six curves as in Fig. 8. In this plot, it is assumed that three anchor nodes participate in the positioning calculation. As it can be seen, with more localization request packets used, the errors can be smaller in certain test positions, such as \# 60. But in contrast, in some other positions, more packets used in calculation leads to worse performance, e.g. test position \# 50. Basically, the general positioning performance when different number of packets are used is comparable.

To further evaluate the over-all performance, the estimation error means based on different number of used RSSI measurement packets are depicted in Fig. 9. Three anchor nodes in each reference point participate calculation. As it can been seen, the best performance is achieved when three localization request packets are used. Even though the differences when different number of packets are used are rather small. More packets used in the calculation do not improve the result. Thus, localization system can achieve acceptable results even without many localization request packets broadcasted. In the practical leverage, the target might merely need to broadcast three to five localization request packets. This is an obvious advantage since the traffic load can be reduced in networking.

- Estimation Error - Number of Involved Anchor Nodes in Each Cluster (Reference Point) During the experiment, we realized that the RSSI differ from each other even the receiver Mulles are deployed quite close to each other. The main contribution to such behavior can be the orientation of antennas between transceivers. Thus we also explore if it is necessary to deploy several anchor nodes (three anchors in our experiment) with different antenna orientation. Fig. 10, 11 and 12 illustrate the performance of positioning assuming one, two and three anchor node(s) is or are retrieving RSSI in each reference point (cluster) respectively. According to the target's realworld amplitudes, the corresponding positioning errors 

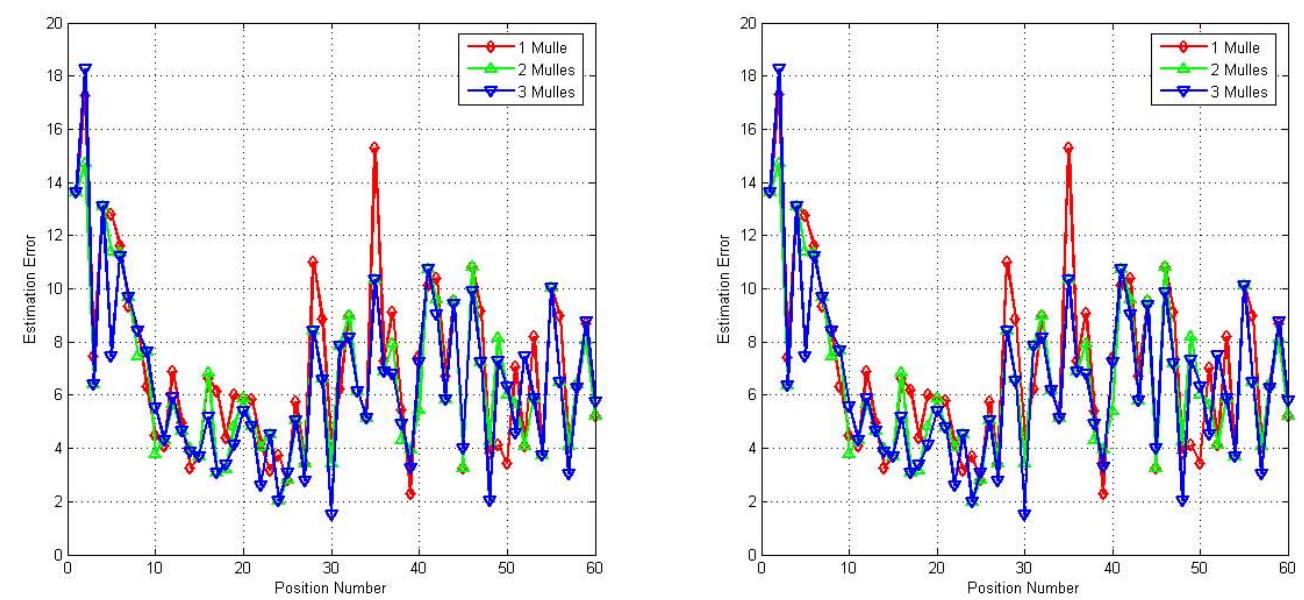

Fig. 7. Five localization request packets and different number of anchors used in each reference point (cluster) with different signal propagation model

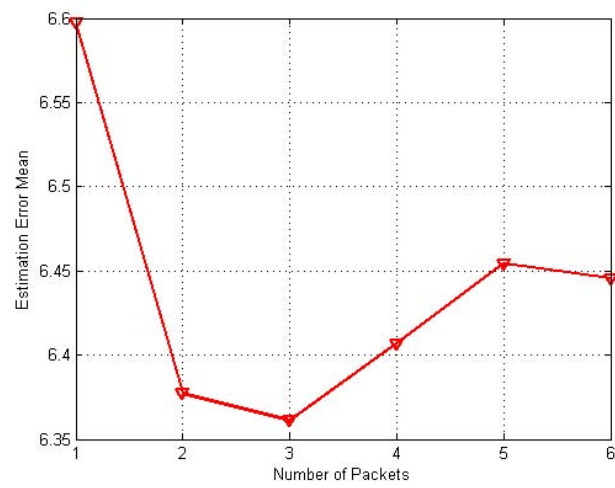

Fig. 9. Mean values of error using different amount of RSSI measurement packets when three anchor nodes in each reference point participate calculation

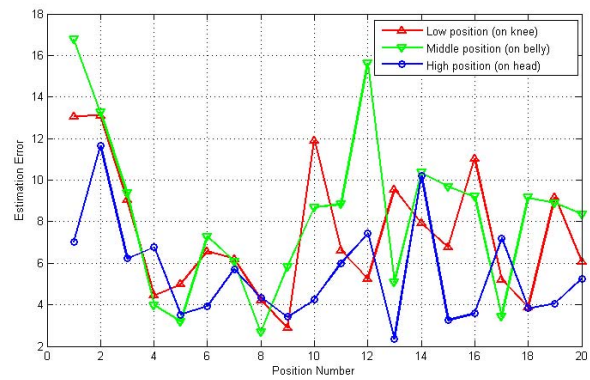

Fig. 10. Errors at different height when using 6 RSSI measurement packets and 1 anchor in each reference point

can be sorted into three groups, red (target is $0.5 \mathrm{~m}$ above ground), green $(1 \mathrm{~m})$ and blue $(2 \mathrm{~m})$. Therefore, the influence of target's height ( $\mathrm{Z}$ axis) on a body is shown in these pictures as well. Basically, positioning is slightly improved by adding anchor nodes into each reference point (cluster). And the position estimation is

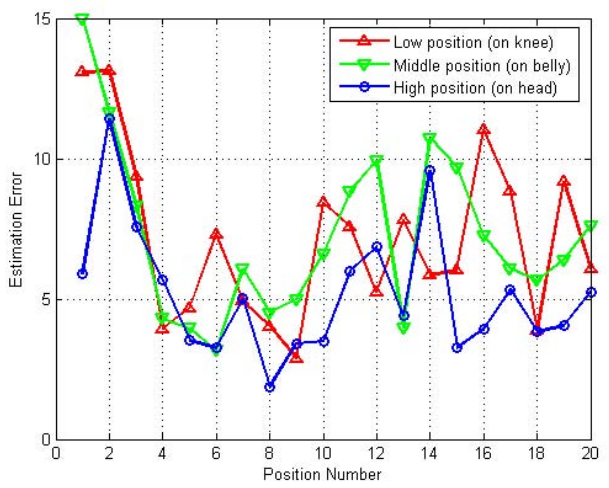

Fig. 11. Errors at different height when using 6 RSSI measurement packets and 2 anchors in each reference point

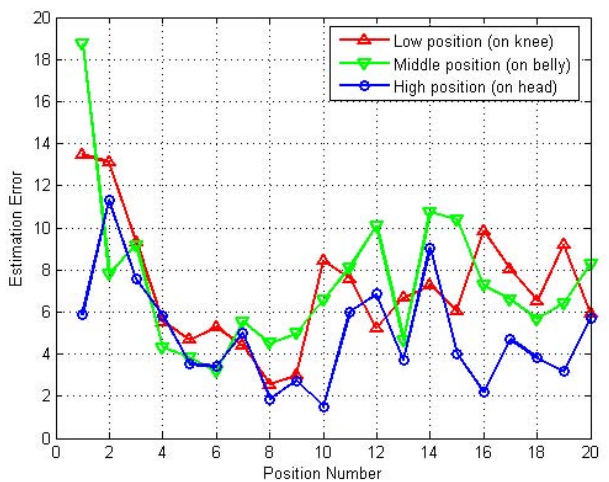

Fig. 12. Errors at different height when using 6 RSSI measurement packets and 3 anchors in each reference point

better when the target is set on head comparing with on knee and belly. Be noticed, the number of RSSI measurement packets used in these calculations is six.

Fig. 13 illustrates the estimation error means for the three 


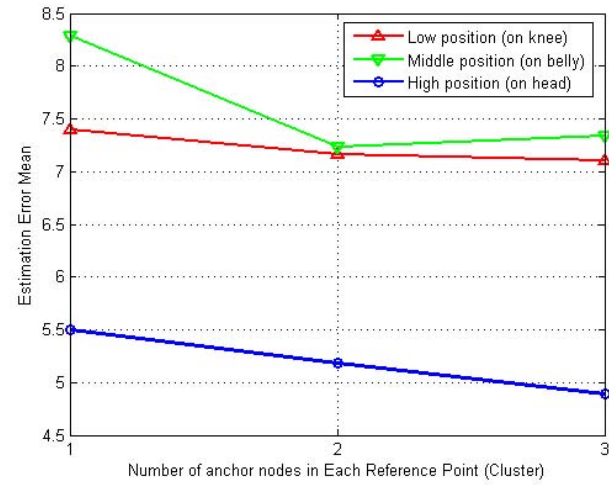

Fig. 13. Error means at different height and using different number of anchors in each reference point with six RSSI measurement packets

error groups against the number of anchor nodes that participate localization in each reference point. For the estimation group when the target is deployed on head, the results are much better than other groups. Generally, more Mulles participating positioning will improve the result slightly.

\section{CONCLUSION}

In this paper, a cluster-based localization method has been presented. The method was evaluated using a new IEEE 802.15.4 based platform, the Mulle v5.2, in a large scale space: $40 \mathrm{~m} * 20 \mathrm{~m} * 6.5 \mathrm{~m}$. The proposed approach is designed to draw from the advantages of a cluster-based sensor network where the signal strength is obtained by having several sensor nodes within a body area sensor network (cluster) collaborate. The network topology consists of a number of inter connected clusters, where each cluster is a body area network. Each BAN is worn by a firefighter in order to remotely monitor his health state and position. By using the cluster approach, a number of devices with differently aligned antennas are equipped on each firefighter. This enables mitigation of the effects of multi-path and obstacles. The proposed localization method is based on a cluster-based version of the MinMax algorithm, which also has the advantage of eliminating the need to transmit a large number of localization request packets. Experiments have shown that different postures of target user will lead to different estimation errors. Potentially, by adding a posture detecting functionality and combining the knowledge of posture with RSSI values, it is expected that the performance of localization method can be further increased. The next step is to investigate if the approach can be used in 3D application scenarios. Tests have shown that the approach can achieve an average error of around 5 meters, which for the present application is sufficiently accurate.

\section{ACKNOWLEDGMENT}

The authors would like to express their gratitude towards the NSS project for funding, and to David Furter for his assistance with the experiments.

\section{REFERENCES}

[1] N. Patwari and A. O. H. III, "Using proximity and quantized rss for sensor localization in wireless networks," in Proceedings of the 2nd ACM international conference on Wireless sensor networks and applications, September 2003.

[2] J. N. Al-Karaki and A. E. Kamal, "Routing techniques in wireless sensor networks: A survey," in Wireless Communications, IEEE, vol. 11, December 2004, pp. 6-28.

[3] N. B. Priyantha, A. Chakraborty, and H. Balakrishnan, "The cricket location-support system," in Proceedings of the 6th annual international conference on Mobile computing and networking, 2000, pp. 32-43.

[4] G. Zanca, F. Zorzi, A. Zanella, and M. Zorzi, "Experimental comparison of rssi-based localization algorithms for indoor wireless sensor networks," in Proceedings of the workshop on Real-world wireless sensor networks, Glasgow, Scotland, 2008, pp. 1-5.

[5] T. Stoyanova, F. Kerasiotis, A. Prayati, and G. Papadopoulos, "Evaluation of impact factors on rss accuracy for localization and tracking applications," in Proceedings of the 5th ACM international workshop on Mobility management and wireless access, New York, 2007, pp. 9-16.

[6] M. Sugano, T. Kawazoe, Y. Ohta, and M. Murata, "Indoor localization system using rssi measurement of wireless sensor network based on zigbee standard," in The IASTED International Conference on Wireless Sensor Networks (WSN 2006), Banff (Canada), July 2006.

[7] E. Elnahraway, X. Li, and R. P. Martin, "The limits of localization using rss," in Proceedings of the 2nd international conference on Embedded networked sensor systems, Baltimore, MD, USA, 2004, pp. 283-284.

[8] X. Nguyen and T. Rattentbury, "Localization algorithms for sensor networks using rf signal strength," in University of California at Berkeley, Tech. Rep., May 2003.

[9] A. Savvides, C.-C. Hand, and M. B. Strivastava, "Dynamic fine-grained localization in ad-hoc networks of sensors," in Proc. 7th international Conference on Mobile Computing and Networking, Italy, 2001, pp. 166179.

[10] R. Parker and S. Valaee, "Robust min-max localization algorithm," in Proc. of the IEEE ITSC 2006, Toronto, Canada, September 17-20 2006.

[11] "Eistec ab." http://www.eistec.se/, 2010.

[12] "At86rf230." http://www.atmel.com/, 2009. 